\title{
STRENGTH OF COMPOSITE YARN UNDER BIAXIAL LOADING
}

\author{
Soňa VAlentová ${ }^{a, *}$, Vladimír HrBeK $^{a, b}$, Jan Vorel $^{a}$, Michal Šejnoha $^{a}$ \\ ${ }^{a}$ Czech Technical University in Prague, Faculty of Civil Engineering, Thákurova 7, 16629 Prague 6, Czech \\ Republic \\ ${ }^{b}$ Institute of Theoretical and Applied Mechanics AS CR, v.v.i., Prosecká 76, 19000 Prague 9, Czech Republic \\ * corresponding author: sona.valentova@fsv.cvut.cz
}

\begin{abstract}
The present paper is concerned with two types of periodic unit cell of composite yarn with different geometry but the same material properties. Their macroscopic response under tensile and compressive loading in the transverse direction and their combination are plotted in the graphs. Based on stress-strain curves the failure envelopes are constructed. A simple maximum stress criterion and linear softening law is used in the adopted progressive damage analysis to outline the softening part of stress-strain diagrams. Finally the impact of selected representative volume element is observed through the comparison of results gained for both designed periodic unit cells in the microlevel, meaning the level of yarn.
\end{abstract}

KEYwORDs: Composite yarn, periodic unit cell, strength, damage analysis, failure envelope.

\section{INTRODUCTION}

Demands on material properties increase as many different industry branches develop. Therefore new materials, like textile composites become popular, especially for their variability, that consist for example in type of material used for fibers and matrix, type of weave, volume fraction of individual phases, curing temperature and so on. Every mentioned factor influences the final macroscopic properties, so one of their big advantage is the possibility to adapt their final macroscopic properties. For their high specific strength, stiffness and temperature resistance they find use for example in automobile or aerospace industry. One of the most typical reinforcement are glass fibers, which belong to the most price favorable fibers and also have quite high modulus and strength. Next commonly used fibers are Carbon and basalt fibers. These are better in terms of mechanical properties. Nowadays carbon fibers are still more often substituted by the basalt fibers thanks to their similar mechanical properties and lower price.

For the possibility an extensive and mainly safe use of such material the knowledge of their behavior under different loading cases for both, in the elastic part and also after the damage occur, is necessary. As the experiments are complicated not only in terms of feasibility, but also from the time and financial point of view, some effective tool to obtain their macroscopic response numerically is desirable.

The structure of braided composite is very complex, thus the appropriate simplification provided through the determination of representative volume element and subsequently construction of periodic unit cell, substituting its structure is important for the numerical analysis.

In this paper, we adopt two types of periodic unit cell in the micro-scale, meaning the level of yarn, with attention concerned to the evaluation of macroscopic strength for unidirectional fiber composite under tensile and compressive on-axis loading in the transverse direction, see Fig. 3 and Fig. 4 and using their combination the failure envelope expressing the effective strengths in arbitrary transverse direction could be draw up.

In case of textile composite, see the cross section and 3D periodic unit cell of such a composite in Fig. 1 for obtaining its macroscopic properties, results from analysis introduced in this work will be applied to the meso-scale level where the effective properties of yarns are needful.

Moreover, the influence of elected periodic unit cell of yarn is investigated via the evaluation of effective elastic properties with the hand of finite element method subsequently compared with elastic properties gained by the Mori-Tanaka averaging scheme.

\section{Periodic Unit Cell of Yarn}

Design of periodic unit cell (PUC) of yarn is based on the image analysis carried out on the cross sections of yarns, see Fig. 2a, consisting of determination of geometrical parameters and then evaluation of volume fractions of individual phases. Based on the knowledge of the inner geometry a representative volume element (RVE) is determined, for more details see [1. Typical and the most simplified PUC of yarn dealing with the idealized hexagonal distribution of fibers was designed, can be seen in Fig. 3 Moreover, 4-fiber PUC of yarn with more complex RVE was created, see Fig. 4, to be able to verify the adequacy of designed PUC. 


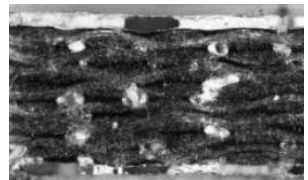

(A).

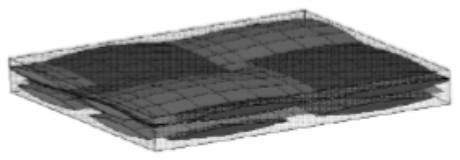

(B).
Figure 1. (A) Cross section of braided composite, (B) 3D-SEPUC of composite.

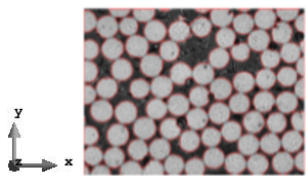

(A).

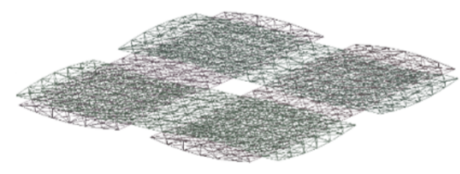

(B).
Figure 2. (A) Cross section of yarns with coordinate system, (B) Example of mesh of yarns in composite.

\section{Failure Criteria and Damage MODEL}

When loading of periodic unit cell representing yarn of braided composite we can distinguish two types of failure modes:

- Fiber failure mode

- Matrix failure mode

In contrast with the direct stiffness reduction method, which simply assign zero stiffness to the integration point of mesh element, where the strength is reached, in adopted progressive damage model proposed by Fang et al. [2, 3] lower individual elements of the stiffness matrix according to the magnitude of loading to reflect a material degradation with the evolution of cracks. Then we can observe the macroscopic response also after the strength is exceeded. Damage evolution is expressed as

$$
D_{J}=\frac{\chi_{e q}^{J f}\left(\chi_{e q}^{J}-\chi_{e q}^{J i}\right)}{\chi_{e q}^{J}\left(\chi_{e q}^{J f}-\chi_{e q}^{J i}\right)}, \quad J=1 T, 2 T, 3 T, 1 C, 2 C, 3 C
$$

In Eq. $1 \chi_{e q}^{J i}$ and $\chi_{e q}^{J f}$ represents the equivalent displacement at the onset of failure and the equivalent displacement at the full damage, respectively. $\chi_{e q}^{J}$ expresses equivalent displacement in the current loading step. The initial value is defined from the first load increment as

$$
\chi_{e q}^{J i}=\frac{\chi_{e q}^{J}}{\sqrt{F_{J}}}
$$

where $F_{J}$ is $J^{\text {th }}$ failure criterion. As a simple maximum stress criterion is adopted in present work, following condition must be fulfilled to start developing the damage after the crack initializes.

$$
\frac{\sigma_{i i}}{S_{i J}}>=1, \quad i=1,2,3 ; \quad J=T, C
$$
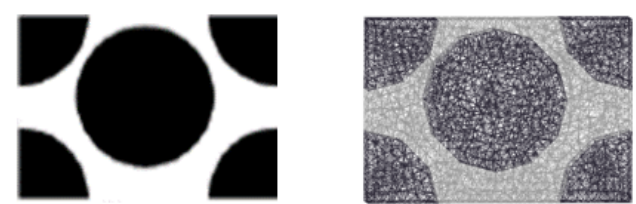

Figure 3. 2-fiber PUC - PUC-1.
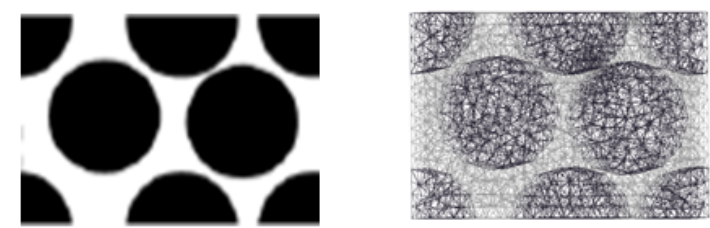

Figure 4. 4-fiber PUC - PUC-2.

To define the displacement at the full damage in the nonlinear constitutive model, the fracture energy as the principal material property is indispensable. It can be written as

$$
\int_{0}^{w_{c}^{f}} \sigma d w_{c}=L_{S} \int_{0}^{f_{d}}\left(\epsilon-\epsilon^{e l}\right) d \sigma=L_{S} \int_{0}^{\epsilon^{f}} \sigma d \epsilon
$$

The fracture energy is graphically expressed in Fig. 5.

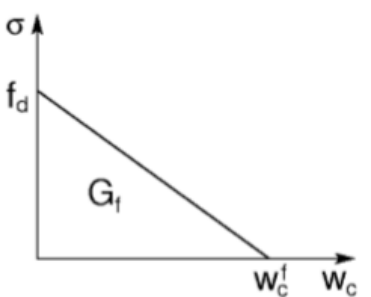

(A).

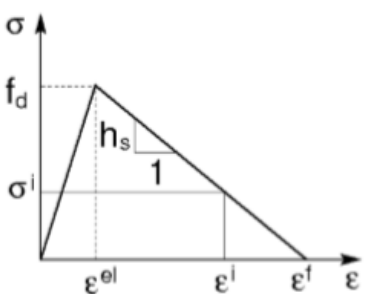

(в).
Figure 5. (A) Stress-crack opening displacement diagram with linear softening law, (B) stress-strain diagram with softening.

\section{Numerical Experiment}

Numerical experiment was carried out on a basalt fiber composite with pyrolized polysiloxane matrix. As the material is heterogeneous, several indentation measurements were performed on both phases of the available sample, fibers and matrix. Results from these measurements are listed in Tab. 1. Average results of elastic material properties partially taken from the experiment, partially from literature, see [4] and partially set according to the analogy with the carbon fibers, are summarized in Tab. 1. Young's modulus was derived from indentation results based on contact mechanics principles of two colliding bodies, as described in [5, 6. These results have already been presented in [7]. Principles of the nanoindantation method are described in [5].

Effective elastic properties of investigated composite summarized in Tab. 3 were also investigated in order to 


\begin{tabular}{|c|c|c|c|c|}
\hline $\begin{array}{c}\text { Measurement } \\
\text { number }\end{array}$ & $\begin{array}{l}\text { Red. mod. } \\
\mathbf{E}_{r}[\mathrm{GPa}]\end{array}$ & $\begin{array}{l}\text { Young's mod. } \\
\text { E [GPa] }\end{array}$ & $\begin{array}{c}\text { Hardness } \\
\text { H [GPa] }\end{array}$ & $\begin{array}{l}\text { Contact depth } \\
\mathbf{h}_{c}[\mathbf{n m}]\end{array}$ \\
\hline \multicolumn{5}{|c|}{ Basalt fiber - longitudinal direction } \\
\hline 1 & $67.81 \pm 8.09$ & $67.72 \pm 7.68$ & $5.27 \pm 0.56$ & $191.92 \pm 6.55$ \\
\hline 2 & $75.95 \pm 6.55$ & $75.66 \pm 6.21$ & $5.95 \pm 0.88$ & $106.09 \pm 8.91$ \\
\hline 3 & $76.85 \pm 7.75$ & $76.63 \pm 7.35$ & $5.71 \pm 0.76$ & $108.33 \pm 7.98$ \\
\hline 4 & $66.17 \pm 10.44$ & $66.08 \pm 9.93$ & $5.49 \pm 0.75$ & $161.55 \pm 12.22$ \\
\hline 5 & $71.76 \pm 7.30$ & $71.15 \pm 6.92$ & $5.79 \pm 0.38$ & $155.99 \pm 5.62$ \\
\hline 6 & $61.12 \pm 6.68$ & $60.85 \pm 6.33$ & $5.28 \pm 0.74$ & $239.19 \pm 18.71$ \\
\hline average & $69.94 \pm 7.80$ & $69.68 \pm 7.40$ & $5.58 \pm 0.68$ & $160.51 \pm 10.00$ \\
\hline \multicolumn{5}{|c|}{ Basalt fiber - transverse direction } \\
\hline 1 & $74.09 \pm 6.22$ & $73.65 \pm 6.22$ & $5.88 \pm 1.17$ & $107.60 \pm 11.80$ \\
\hline 2 & $75.98 \pm 3.79$ & $75.69 \pm 3.58$ & $5.94 \pm 0.54$ & $105.58 \pm 5.57$ \\
\hline 3 & $57.31 \pm 3.58$ & $56.85 \pm 3.38$ & $5.00 \pm 0.55$ & $116.33 \pm 7.25$ \\
\hline 4 & $55.34 \pm 10.55$ & $54.80 \pm 10.03$ & $4.45 \pm 1.16$ & $126.64 \pm 17.08$ \\
\hline 5 & $68.77 \pm 8.09$ & $68.65 \pm 7.68$ & $5.15 \pm 0.54$ & $114.39 \pm 6.80$ \\
\hline 6 & $59.61 \pm 11.70$ & $59.26 \pm 9.44$ & $5.29 \pm 1.22$ & $117.95 \pm 15.13$ \\
\hline average & $65.18 \pm 7.32$ & $64.82 \pm 7.03$ & $5.29 \pm 1.19$ & $114.52 \pm 14.15$ \\
\hline \multicolumn{5}{|c|}{ Pyrolyzed polysiloxane matrix } \\
\hline 1 & $67.57 \pm 3.82$ & $67.47 \pm 3.61$ & $7.29 \pm 0.96$ & $56.64 \pm 4.25$ \\
\hline 2 & $56.45 \pm 3.77$ & $55.95 \pm 3.56$ & $5.44 \pm 0.44$ & $110.65 \pm 4.90$ \\
\hline 3 & $60.20 \pm 5.09$ & $59.88 \pm 4.82$ & $6.01 \pm 0.54$ & $104.88 \pm 5.21$ \\
\hline 4 & $56.70 \pm 8.53$ & $56.22 \pm 8.10$ & $5.63 \pm 1.02$ & $109.81 \pm 10.39$ \\
\hline 5 & $58.39 \pm 7.29$ & $57.98 \pm 6.91$ & $5.68 \pm 0.99$ & $109.45 \pm 11.72$ \\
\hline 6 & $55.82 \pm 5.00$ & $55.30 \pm 4.73$ & $6.23 \pm 1.08$ & $103.85 \pm 10.21$ \\
\hline average & $59.19 \pm 5.58$ & $58.83 \pm 5.29$ & $6.05 \pm 0.84$ & $99.21 \pm 7.78$ \\
\hline
\end{tabular}

TABLE 1. Indentation results of individual material phases.

\begin{tabular}{lcccccc}
\hline Material & $\mathbf{E}$ & $\mathbf{G}$ & $\nu$ & $\mathbf{G}_{f}$ & $\mathbf{S}_{T}$ & $\mathbf{S}_{C}$ \\
& {$[\mathbf{G P a}]$} & {$[\mathbf{G P a}]$} & {$[-]$} & {$[\mathbf{N} / \mathbf{m m}]$} & {$[\mathbf{G P a}]$} & {$[\mathbf{G P a}]$} \\
\hline Fiber - longitudinal direction & 69.68 & 28.10 & 0.40 & - & 2 & -1.5 \\
Fiber - transverse direction & 64.82 & 26.14 & 0.24 & - & 0.5 & -1.5 \\
Pyrolyzed polysiloxane matrix & 58.83 & 23.72 & 0.24 & 0.001 & 0.08 & -0.16 \\
\hline
\end{tabular}

TABle 2. Considered parameters of individual materials.

verify the adequacy of selected representative volume elements. They were obtained by the micromechanical Mori-Tanaka averaging scheme, which builds upon the knowledge of the shape and orientation of the reinforcement, material properties and volume fraction of individual phases. Next, they were gained with the help of more complex first order homogenization approach, but on the other hand more numerical and time demanding method with the need of construction of PUC.

The elastic properties provided by the finite element method for two designed PUCs illustrated in Fig. 3 and Fig. 4 agrees well with the Mori-Tanaka predictions.

The main part of presented paper is numerical evaluation of strength of mentioned composite. Such an analysis was performed in the transverse direction of both designed PUCs. At first both the on-axis tensile and compressive loading and then their combinations necessary for construction of the failure envelope were applied. The failure envelope plotted in the Fig. 10 illustrates the strength in the transverse direction of yarn. The shape perfectly corresponds to our presumption leading from the adopted simple maximum stress criterion mentioned above in Eq. 3. The maximum and minimum values of reached effective stresses are marked and provided in the graphs for the on-axis loading directions.

Further and the last output of this study is evaluation of the nonlinear macroscopic response of the composite. For this purpose, stress-strain curves for both PUCs were generated (Fig. 6 to Fig. 9). One for each on-axis transverse direction for tensile and compressive loading increments. 


\begin{tabular}{lccccccc}
\hline Model type & $\begin{array}{c}\mathbf{E}_{11} \\
{[\mathbf{G P a}]}\end{array}$ & $\begin{array}{c}\mathbf{E}_{22} \\
{[\mathbf{G P a}]}\end{array}$ & $\begin{array}{c}\mathbf{E}_{33} \\
{[\mathbf{G P a}]}\end{array}$ & $\begin{array}{c}\mathbf{G}_{23} \\
{[\mathbf{G P a}]}\end{array}$ & $\begin{array}{c}\mathbf{G}_{31} \\
{[\mathbf{G P a}]}\end{array}$ & $\begin{array}{c}\mathbf{G}_{12} \\
{[\mathbf{G P a}]}\end{array}$ & $\begin{array}{c}\mathbf{c}_{f} \\
{[-]}\end{array}$ \\
\hline SEPUC-1 - FEM & 62.96 & 62.97 & 65.86 & 26.46 & 26.48 & 23.5 & 0.62 \\
SEPUC-2 - FEM & 62.98 & 62.96 & 65.86 & 26.48 & 26.46 & 23.5 & 0.62 \\
Mori-Tanaka method & 62.97 & 62.97 & 65.88 & 26.47 & 26.47 & 23.5 & 0.62 \\
\hline
\end{tabular}

TABLE 3. Effective elastic moduli.

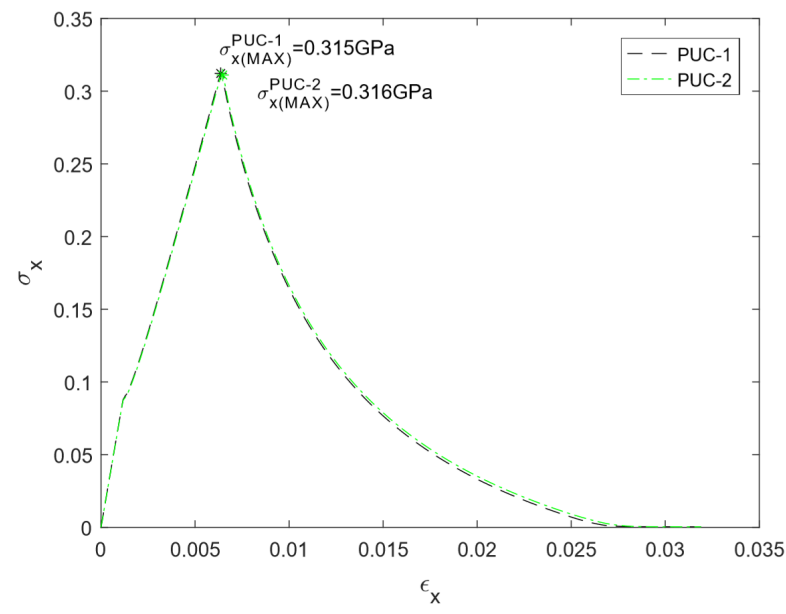

Figure 6. Stress-strain curves for on-axis tensile loading in the $\mathrm{x}$-direction.

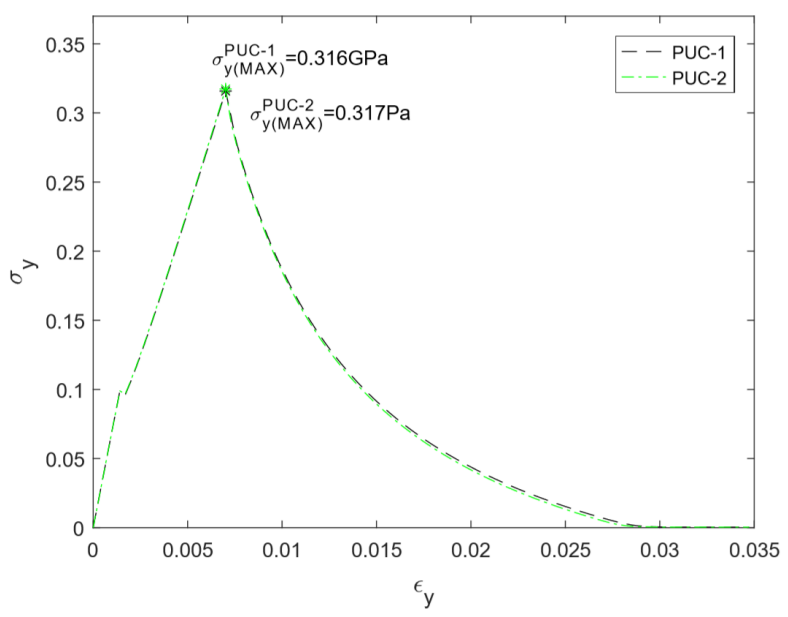

FiguRE 7. Stress-strain curves for on-axis tensile loading in the y-direction.

Evidently the macroscopic response gained for both PUCs gives almost the same results not only in the elastic part but also for the nonlinear behavior with just negligible differences.

\section{Conclusion}

The presented paper was concerned with two types of periodic unit cell of composite yarn with different representative volume element but the same material properties. At first their effective elastic properties were obtained both by the Mori-Tanaka averaging scheme and finite element method. Based upon gained

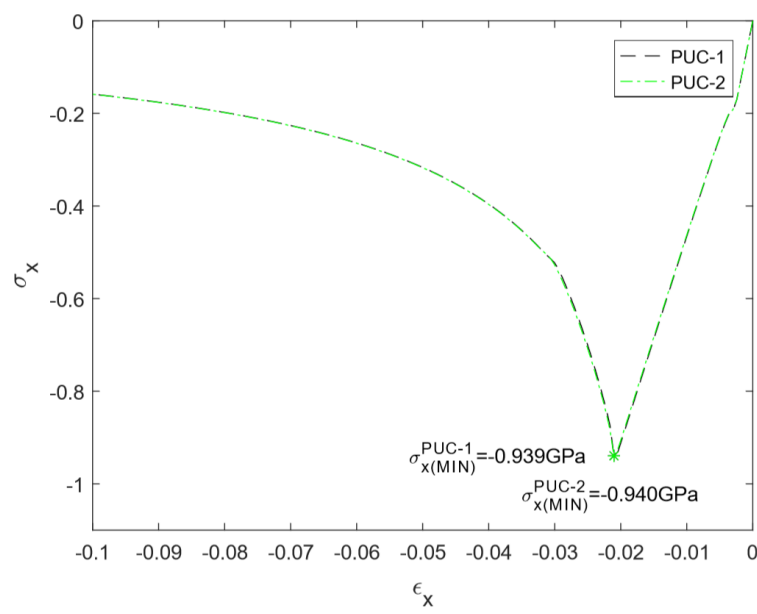

FiguRE 8. Stress-strain curves for on-axis compressive loading in the $\mathrm{x}$-direction.

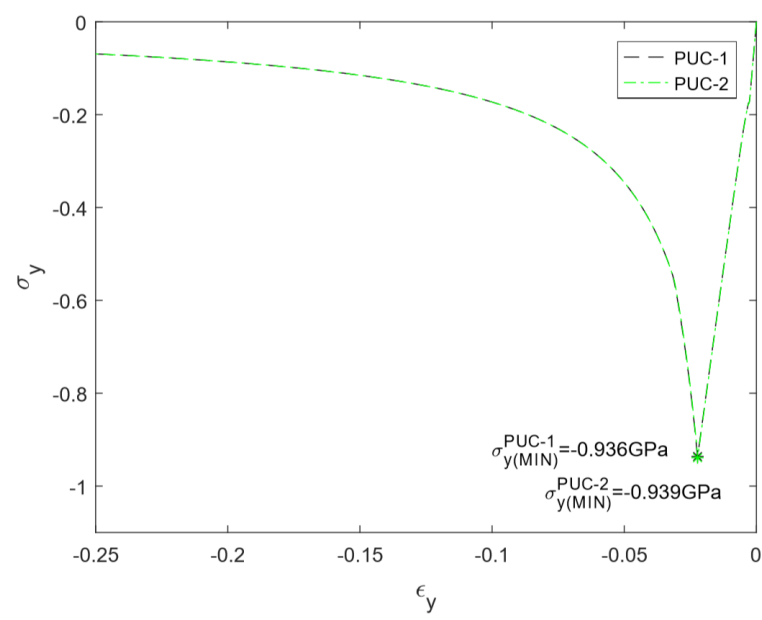

FiguRE 9. Stress-strain curves for on-axis compressive loading in the y-direction.

results the adequacy of the designed PUC could be verified.

In the next step the macroscopic elastic and nonlinear response for tensile and compressive loading in the transverse on-axis direction were investigated and plotted in the diagrams. On the basis of the presented methodology we can observe macroscopic behavior of composite after cracks initialize and damage starts to develop. A simple maximum stress criterion and linear softening law was adopted in this study to outline the nonlinear part of stress-strain diagrams. Based on the stress-strain curves derived from the biaxial loading, the failure envelopes were constructed. Re- 


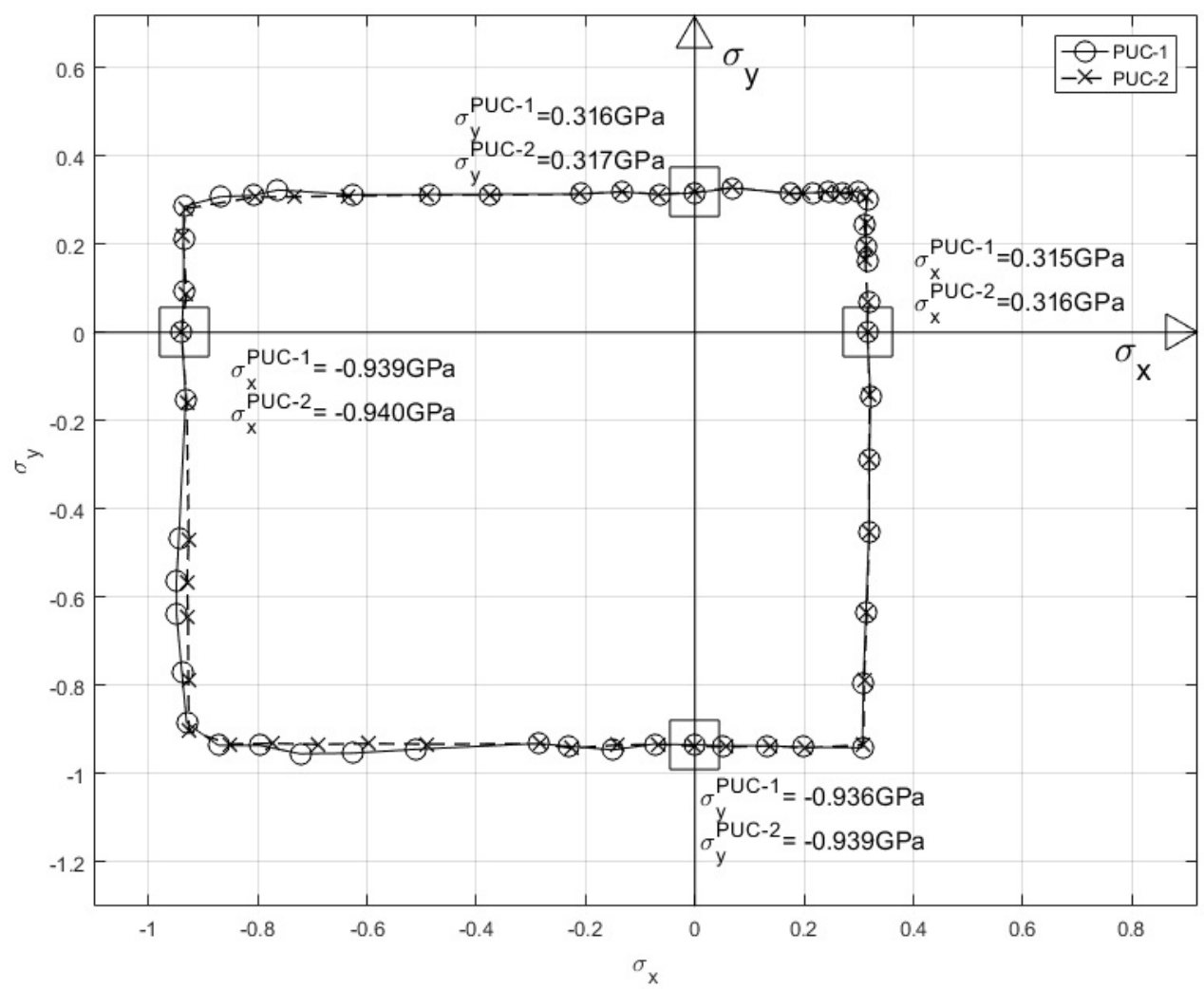

FiguRE 10. Failure envelope for biaxial loading in the transverse direction.

alized computational analysis shows the macroscopic strength of investigated composite for the input parameters summarized in Tab. 3 Differences in failure envelopes (Fig. 10) and in stress-strain curves raised for both examined PUCs (Fig. 6 to Fig. 9 ) are almost imperceptible and could be caused by the unequal generated mesh density. Consequently we can afford to consider the disparities such insignificant that the simplification of yarn through the designed PUC is perfectly acceptable.

The model was tested on mentioned composite but with incomplete knowledge of needed input material parameters. In the future work we will pay our attention to another numerical and nanoindentation experiments to get necessary parameters to accomplish more accurate analysis on micro-scale and continue with numerical predictions of composite with more complex structure, e.g. illustrated in Fig. 1

\section{LIST OF SYMBOLS}

$\nu$ Poisson ratio $[-]$

$E_{r}$ Reduced modulus [GPa]

$E$ Young's modulus [GPa]

$H$ Hardness [GPa]

$h_{c}$ Contact depth [nm]

$G$ Shear modulus [GPa]

$G_{f}$ Fracture energy [N/mm]

$S_{T}$ Critical strength in tension [GPa]
$S_{C}$ Critical strength in compression [GPa]

$E_{i j}$ Homogenized elastic modulus [GPa]

$G_{i j}$ Homogenized shear modulus [GPa]

$D_{J}$ Damage parameter [-]

$\chi_{e q}$ Equivalent displacement [m]

$\sigma_{i j}$ Stress [GPa]

$\epsilon_{i j}$ Strain [-]

\section{ACKNOWLEDGEMENTS}

The financial support provided by the SGS project with the application registered under the No. SGS18/037/OHK1/1T/11 is gratefully acknowledged.

\section{REFERENCES}

[1] J. Zeman, M. Šejnoha. From random microstructures to representative volume elements. Modelling and Simulation in Materials Science and Engineering 15(4):325-335, 2007. DOI:10.1088/0965-0393/15/4/S01.

[2] G. D. Fang, J. Liang, Q. Lu. Investigation on the compressive properties of the three dimensional four-directional braided composites. Composite Structures 93(2):392-405, 20011. DOI:10.1016/j.compstruct.2010.09.002

[3] G. D. Fang, J. Liang, B. L. Wang. Progressive damage and nonlinear analysis of $3 \mathrm{~d}$ four-directional braided composites under unidirectional tension. Composite Structures 89(1):126-133, 20009. DOI:10.1016/j.compstruct.2008.07.016 
[4] M. Černý, P. Glogar, Z. Sucharda. Mechanical properties of basalt fiber reinforced composites prepared by partial pyrolysis of a polymer precursor. Journal of Composite Materials 43(9):1109-1120, 2009. DOI:10.1177/0021998308097732

[5] W. C. Oliver, G. M. Pharr. Measurement of hardness and elastic modulus by instrumented indentation: Advances in understanding and refinements to methodology. Journal of Material Research 19(1):3-20, 2004. DOI:10.1557/jmr.2004.0002
[6] H. Hertz. On the contact of rigid elastic solids. Hertz's Miscellaneous Papers p. Chap. 6, 1896. Original: Über die Berührung fester elastischer Körper, Journal für die Reine und angewandte Mathematik, 92: 156-171, 1881.

[7] S. Valentová, V. Hrbek, M. Šejnoha. Evaluation of effective properties of basalt textile reinforced ceramic matrix composite. Acta Polytechnica CTU Proceedings 13:142-147, 2017. DOI:10.14311/APP.2017.13.0142 